Convergences francophones 3.1 (2016) : 56-70

http://mrujs.mtroyal.ca/index.php/cf/index

\title{
Ailleurs, au Mexique : topographie géopoétique d'Ourania de J.-M.G. Le Clézio
}

\author{
Rachel Bouvet \\ Université du Québec à Montréal
}

\section{Penser l'ailleurs}

Comment penser l'ailleurs en littérature? Si l'on prend comme point de départ le langage et la discipline qui l'étudie, à savoir la linguistique, qui a longtemps servi de cadre de référence pour les études littéraires, on peut envisager l'ailleurs comme un espace absent. La théorie de l'énonciation met en effet l'accent sur l' " ici », le lieu où se trouve l'énonciateur, et le met en rapport avec « là-bas », cet endroit que l'on peut pointer du doigt, un lieu se trouvant à une certaine proximité, physique et/ou affective; le couple ici/là-bas se superpose au couple je/tu, à la base des interactions humaines (Benveniste). Ici / là-bas / ailleurs : la gradation se fait du plus connu au moins connu, de la présence à l'absence. Ailleurs, c'est l'endroit que l'on ne connaît pas, un lieu dont on rêve ou qui nous fait peur, un lieu dont on parle sans y être allé, c'est l'espace absent de l'énonciation, tout comme le « il» ou le «elle» sont les pronoms relatifs aux personnes absentes de la situation d'énonciation.

Que faire lorsqu'il ne s'agit pas d'un dialogue entre deux interlocuteurs mais d'un texte écrit ? Étant donné que les déictiques (pronoms, embrayeurs) se rapportent à un sujet énonciateur ou à un narrateur, on a tendance à définir l'ailleurs par rapport aux instances narratives. Doit-on pour autant s'arrêter aux limites du texte ? Que dire de la lecture des traductions, une pratique qui a tendance à se multiplier? Si le lecteur ne connaît pas la culture dont le texte est issu, il aura de la difficulté à se représenter les lieux, qui se situent pour lui dans un « ailleurs ». Par exemple, l'écrivain nobélisé Naguib Mahfouz représente dans ses romans un espace qu'il connaît bien, qui lui est familier et qui est pour lui - de même que pour ses narrateurs et personnages - de l'ordre de l' « ici », mais le lecteur ne connaissant pas l'Égypte du XX $\mathrm{XX}^{\mathrm{e}}$ siècle a l'impression d'être immergé dans un espace exotique, d'être transporté ailleurs, dans un univers qui lui est complètement étranger. La traduction suscite des effets d'étrangeté plus ou moins forts suivant le mode qu'elle utilise, mais dans tous les cas, la perspective du lecteur doit être prise en compte pour pouvoir définir l'ailleurs (Bouvet Notes de traduction). En s'interrogeant sur les caractéristiques du processus de lecture, les théories de la lecture et de la réception qui se sont développées depuis les années 80 ont permis de mettre à jour certaines caractéristiques de la production de signification et de s'interroger sur la façon dont le lecteur se représente l'espace du récit. Comme Victor Segalen l'avait bien remarqué en réfléchissant à la question de l'exotisme, l'ici et l'ailleurs ont cette faculté d'échanger leur place dès qu'un déplacement se fait, dans un sens ou dans l'autre : c'est ce qu'il appelait la « réversibilité de l'exotisme ».

Une autre avenue possible consiste à s'intéresser aux auteurs qui se sont rendus «ailleurs» (comme Segalen justement) et qui en ont rapporté des 
témoignages. Le cas du récit de voyage est particulièrement intéressant pour la problématique de l'ailleurs puisqu'il a pour trame principale un trajet allant de l'« ici » (du voyageur) à l'ailleurs. Les études sur le genre viatique accordent ainsi une place importante aux diverses formes d'interaction avec l'espace de l'ailleurs, un espace défini avant tout par son altérité, à la fois sur le plan humain et sur le plan culturel (Holtz \& Masse).

Cela dit, l'intérêt récent pour les rapports entre littérature et géographie a suscité un ensemble de réflexions sur l'espace qui permettent de penser l'ailleurs autrement. La perspective transdisciplinaire de la géopoétique apparaît novatrice à cet égard (Lévy). Fondée par le poète et essayiste Kenneth White, la géopoétique se présente comme un champ de recherche et de création transdisciplinaire cherchant à développer un rapport au monde à la fois sensible et intelligent. Débordant de loin le cadre de la littérature, puisqu'elle implique un cheminement individuel et un questionnement d'ordre existentiel, elle peut donner lieu à une approche du texte littéraire basée sur certains principes importants comme le dehors, le mouvement et la critique radicale. La lecture géopoétique des textes tire profit de certaines notions étudiées en géographie, telles que le paysage, la mobilité, la carte et l'habiter, pour déployer la signification du texte et offrir au lecteur la possibilité de transformer son regard sur le monde (Bouvet Vers une approche). Les textes dans lesquels l'ailleurs se trouve représenté forment une catégorie intéressante à examiner d'un point de vue géopoétique dans la mesure où le rapport à l'ailleurs engage un rapport au monde. Se pose alors la question des relations entre l'ailleurs et l'écriture: comment nommer l'ailleurs ? Quels sont les outils du géographe et de l'écrivain ? Le roman Ourania (2006) de J.-M.G. Le Clézio offre un éclairage intéressant pour répondre à ces questions parce qu'il met en scène un géographe aux prises avec l'ailleurs. Le protagoniste, Daniel Sillitoe, se rend au Mexique afin de faire des relevés pédologiques, mais il laisse de côté momentanément sa mission pour découvrir le pays. La présence de ce géographevoyageur (Ballot) donne au lecteur l'occasion de construire l'espace de la fiction par le biais d'un filtre tout à fait singulier.

Si les romans lecléziens ont parfois été qualifiés de " romans géographiques » (Moura), on peut dire que ce genre atteint son acmé avec Ourania. Le fait que le narrateur-personnage soit un géographe en mission dans un autre continent joue un rôle déterminant dans la diégèse, bien évidemment, mais il importe de préciser que ce géographe a la "passion du monde », pour reprendre une expression utilisée pour parler d'Élisée Reclus, un autre géographe du XIX ${ }^{\mathrm{e}}$ siècle (Eprendre). Sa curiosité n'a d'égale que sa propension à passer "d'un monde à un autre ». Je commencerai par observer le mouvement général du récit avant d'examiner plus spécifiquement ce qui relève de la géographie, en particulier le discours et le parcours de Daniel Sillitoe, afin de comprendre dans quelle mesure il appréhende l'ailleurs à partir d'un « œil géographique » (Waddell). Ensuite, je me livrerai à une topographie du roman à partir de l'examen des toponymes, des patronymes et des prénoms, qui sont ici les outils privilégiés pour évoquer l'ailleurs. Cela donnera l'occasion d'interroger le rapport entre l'écriture et le lieu.

\section{Passer d'un monde à un autre monde}


Le premier chapitre a pour cadre la maison familiale durant la guerre, un monde fermé, à l'horizon bouché par le papier bleu que l'on pose le soir sur les vitres, un monde plein d'odeurs dans lequel les seules échappées possibles sont offertes par les livres. C'est pour fuir le monde réel que l'enfant invente un pays imaginaire : « ... du livre rouge sortaient des mots, des noms. Chaos, Éros, Gaia et ses enfants, Pontos, Océanos et Ouranos le ciel étoilé. ... Je ne connaissais que les dessins de la toile cirée, l'odeur de soufre, et la voix chantante de ma mère qui lisait » (Le Clézio Ourania, 18). Véritable support à la rêverie, la toile cirée joue le rôle d'une carte - rappelons que « carte " vient du latin « charta », papier, mais qu'il s'agit plus généralement d'un support pour les signes et que toutes sortes de matériaux sont utilisés pour dessiner des cartes (Jacob). Les mots lus par la mère, associés aux motifs de la toile cirée, créent une piste d'envol pour les rêves de l'enfant. Le pays inventé ne se superpose pas au monde extérieur, que l'enfant ne peut explorer en raison de la guerre ; il l'efface pour lui substituer un autre monde.

Ce mouvement consistant à passer d'un monde à un autre se retrouve dans le chapitre suivant, où l'on suit Daniel, devenu adulte, en voyage au Mexique. Surpris par l'étrangeté de son jeune voisin dans le car, il la met d'abord sur le compte de son expression, grave et franche, et de la fascination qu'exerce sur lui le paysage désertique défilant par la fenêtre. Quand le jeune homme lui dit d'où il vient, le nom de Campos déclenche un silence lourd de sens :

Nous sommes restés un long moment sans rien nous dire. Le paysage catastrophique de la sierra volcanique transversale lançait des éclairs blancs à travers la glace teintée. En contrebas, j'aperçus en un coup d'œil le lit du fleuve Armeria, puis le car a commencé à rouler dans une plaine monotone, poudreuse, et je pensais au décor des livres de Rulfo, à Comala pareille à une plaque de fer chauffée à blanc par le soleil, où les humains sont les seules ombres vivantes.

C'était un pays inquiétant, un pays pour aller d'un monde à un autre monde (Le Clézio Ourania 27)

Plusieurs éléments méritent d'être soulignés dans cette citation. D'abord, l'attention se tourne vers l'extérieur, vers le paysage volcanique qui sera l'un des paysages les plus importants du roman: celui-ci lance "des éclairs blancs », comme s'il voulait exprimer quelque chose, et se transforme ensuite pour prendre les traits d'un paysage désertique, ce que l'on devine à partir des termes « plaine monotone, poudreuse » et de l'image de la « plaque de fer chauffée à blanc par le soleil », une perception visuelle qui convoque d'emblée un paysage littéraire. Daniel reconnaît les lieux pour les avoir déjà «lus» sous la plume d'auteurs mexicains comme Juan Rulfo ; autrement dit, les mots jouent le rôle de filtre dans la contemplation du paysage. L'impression qui domine dans cette perception se trouve résumée dans la dernière phrase : il s'agit d'un "pays inquiétant», au caractère démesuré, flou (les humains ne sont que des ombres) et indéterminé, autant de qualités qui le rendent susceptible d'ouvrir un passage vers un autre monde. Enfin, ce n'est sans doute pas par hasard si cette contemplation silencieuse a lieu juste après que le nom de Campos a été prononcé. Ce toponyme attise la 
curiosité de Daniel, qui à partir de ce moment tâchera d'en savoir plus sur le village. Le jeune Raphaël jouera le rôle d'intermédiaire avec cet " autre monde » qui se dessine à l'horizon, un ailleurs inaccessible pour Daniel, à qui l'entrée dans le village ne sera jamais permise. Seuls les manuscrits laissés par le jeune métis ouvriront la piste de Campos, qui conservera jusqu'à la fin une bonne partie de son mystère.

Le trajet est donc similaire à celui qui consistait à inventer un pays imaginaire dans le premier chapitre : en effet, puisque Daniel se borne à rêver à partir des lettres de Raphaël, et à le suivre ici et là sans jamais pénétrer dans l'enceinte du village, on peut dire que le trajet va du réel à l'imaginaire; sauf que dans ce cas, on assiste à une intrication beaucoup plus complexe entre la géographie réelle, celle du Mexique, et les lieux imaginaires. Le trajet va aussi de la terre au ciel, comme dans le cas du pays inventé durant l'enfance. Le paysage volcanique inquiétant ouvre la voie à un monde dans lequel les repères principaux sont ceux de la voûte céleste : en effet, les habitants de Campos sont associés à une famille de divinités provenant des panthéons mythologiques les plus divers et ils reçoivent chacun un morceau de la carte du ciel. Ce mouvement de la terre vers le ciel se trouve d'ailleurs bien illustré par les trois cartes présentées en annexe : la carte de l'ouest mexicain, le plan de Campos et la carte du ciel. Comme le fait remarquer Isabelle Roussel-Gillet dans un article sur « Les cartes du ciel à l'œuvre chez Le Clézio ", le ciel ne parvient jamais à devenir territoire : "Impossible cielitoire, le ciel "n'a pas de frontières" (Le Clézio L'Inconnu 293); la présence d'un ciel étoilé rompt les frontières du corps et du cosmos » (Les cartes du ciel 6). En définitive, autant pour les cartes que pour les espaces privilégiés par le récit, une progression similaire peut être observée, qui va de la terre jusqu'au ciel en passant par un village « fait de songes », dont l'existence est pourtant affirmée.

L'attirance pour cet autre monde, dont le caractère utopique a fait l'objet de plusieurs études (Salles, Thibault, Cavallero), ne tend pas à effacer pour autant le réel. La curiosité est aussi attisée par le voyage au Mexique et c'est une véritable «passion du monde» qui se dévoile à travers le récit. Pour reprendre une expression utilisée par Éric Waddell, celui-ci révèle un « œil géographique » autant par le biais du discours du géographe que par son attitude envers la Terre.

\section{Le discours du géographe}

Le moment fort de la carrière du géographe a lieu au moment où il prononce sa conférence à l'Emporio intitulée « Pedologia - El retrato de la tierra (Pédologie - le portrait de la terre)», devant un public composé de chercheurs - historiens et anthropologues principalement -, et de gens du coin - notaires, médecins, employés de banque, ouvriers agricoles, fermiers. Cette conférence, dont le texte s'étale sur huit pages (Ourania 74-82), prend la forme d'une cosmogonie dans la mesure où Daniel raconte l'histoire de la région en remontant à l'émergence des volcans : « Je parlais de la naissance de leur pays, des volcans qui avaient vomi leur lave et leur cendre, ces volcans pareils à des dieux, le Nevado de Colima, le Tancitaro, le Patamban, Xanoato Jucatzio, ... » (77). L'apparition d'une faille, d'un fleuve, la descente des glaciers, bref l'histoire du monde passionne l'orateur au plus haut 
point : « mes mots résonnaient comme ceux d'une poésie » (76), « je sentais monter en moi l'ivresse » (76). Le conférencier passe en revue tous les âges de la terre, depuis la naissance des volcans jusqu'à l'apparition de l'homme; puis il rappelle les premiers âges nomades, la composition des sols, la germination des plantes indigènes - la tomate et le maïs par exemple -, leur migration vers d'autres continents. Il insiste sur l'arrivée d'un fruit en provenance d'Asie et d'Europe, le fraisier, un fruit jouant un rôle déterminant dans l'économie et la politique et gouvernant l'ensemble des relations humaines dans la vallée: source d'enrichissement pour les uns, c'est aussi une source de malheur pour les autres, pour les enfants notamment - il suffit de penser à « la douleur des petits doigts des enfants que l'acide des fraises ronge jusqu'au sang, jusqu'à faire tomber leurs ongles » (80). En plus de dénoncer la cupidité, il tente de sensibiliser son auditoire aux dangers de la monoculture de la fraise. Ses déclarations font alors vibrer la fibre écologiste. L'un des moyens rhétoriques qu'il utilise consiste à associer la terre, le sol, à la peau de l'être humain : « la terre est notre peau. Comme notre peau, elle change, elle vieillit, elle s'affine ou s'endurcit selon les traitements qu'elle reçoit, elle se craquelle, elle se blesse » (81). Au fur et à mesure que le discours se déploie, la métaphore organiciste (Berdoulay) acquiert des traits plus spécifiques, associés à ceux d'une femme indienne :

En faisant [ce portrait de votre Vallée], il me semblait que je peignais pour vous le corps d'une femme, un corps vivant à la peau sombre, imprégné de la chaleur des volcans et de la tendresse des pluies, un corps de femme indienne plein de force et de jeunesse. Prenez garde à ce que ce corps de femme si beau et généreux ne devienne, du fait de votre âpreté au gain ou de votre inconscience, le corps desséché et stérile d'une vieille à la peau grise, décharnée, vouée à la mort prochaine (82)

Cette alliance entre la terre et le corps féminin se retrouve comme chacun le sait dans la majorité des métaphores organicistes, aussi bien celles qui émaillent le discours ordinaire que celles que l'on trouve dans la littérature (Bureau). Mais ce qu'elle a ici de particulier, c'est qu'elle se trouve à l'origine même de la fascination pour Lili de la lagune, cette jeune fille « venue du fond des montagnes, de Yalalag, d'Oaxaca » (107), une jeune fille semblable à une fleur, « une fleur indienne, la fleur de mai par exemple, avec ses pétales veloutés, son parfum de vanille et de poivre, une fleur éclatante de jeunesse et de vie » (110), une jeune fille possédant un " corps de femme et [un] visage d'enfant», un " parfum de terre » (110). Les « rois de la fraise » et les patrons des usines, tous ceux qui exploitent le sol à outrance, abusent également des filles de la Zone comme Lili. Celles-ci perdent leur jeunesse et leur force en vivant dans un état d'humiliation et de servitude des plus atroces. Les anthropologues de l'Emporio eux-mêmes, sous couvert de recherches scientifiques, n'hésitent pas à posséder la jeune prostituée, ce qui choque Daniel au plus haut point. Épuisé par les mauvais traitements, à l'instar du sol, le corps de Lili est en passe de se dessécher à l'image de la vieille femme près de laquelle elle vit.

Daniel s'offusque de l'attitude des exploiteurs, qui ont réduit son corps en esclavage, et dénonce le comportement des anthropologues, qui en ont fait un objet 
d'étude. Il s'inscrit quant à lui dans une autre lignée masculine, celle des géographes passionnés par le monde et par les mots : son grand-père paternel était professeur d'histoire-géo avant de se consacrer au mysticisme; lui-même est devenu professeur d'histoire-géo au Collège Alphonse-Allais de Blainville en Seine-Maritime ; ces mentions suffisent à elles seules pour que surgisse l'ombre de Julien Gracq, le plus célèbre des écrivains-géographes français, ce que les critiques n'ont pas manqué de souligner (Van Acker, Rondeau); lui aussi enseignait l'histoire-géo au lycée, en Normandie notamment. Au moment de choisir le sujet de la conférence, le professeur Don Thomas cite Humbolt, souvent considéré comme le père de la géographie, et Lumholtz, un explorateur et ethnographe norvégien : «Vous pourriez parler de Humboldt, ou de Lumholtz, l'auteur du Mexico desconocido, vous savez qu'il est passé par ici, [...] avant de s'aventurer dans la sierra tarasque. Il s'était mis en tête de rapporter à la société géographique de New York le cadavre momifié d'un Indien ... ça a failli lui coûter la vie ...» (62). Un peu plus tard, avant que Daniel parte en expédition dans la vallée du Tepalcatepec, il lui fait une confidence: «Le Mexique est la terre rêvée des géographes ... Vous suivrez la trace de Lumhotz » (226) D'ailleurs, dans le dernier chapitre, au moment où Daniel entame son "deuxième voyage de géographe ", vingt-cinq ans après son premier séjour au Mexique, en direction du Belize cette fois, l'idée de s'adresser à une société géographique surgit : «Peut-être ferai-je une conférence sur la deuxième plus grande barrière de corail du monde, celle du Belize, pour la Société géographique de Rouen, en souvenir de l'explorateur Camille Douls » (275). Les allusions à Camille Douls, cet aventurier un peu fou mort à l'âge de vingt-cinq ans lors de son deuxième séjour au Sahara, qu'il avait traversé en étant déguisé en musulman, sont fréquentes chez Le Clézio. Celui-ci a d'ailleurs signé l'introduction de la biographie de ce personnage au destin exceptionnel (Roussanne). À son retour du désert, Douls avait prononcé des conférences dans plusieurs Sociétés de géographie, à Rouen notamment, et son récit de voyage avait été publié par la Société géographique de Paris. En formulant le souhait de contribuer à la connaissance des fonds marins béliziens, Daniel Sillitoe fait plus que suivre la trace d'éminents géographes-voyageurs, il boucle la boucle en inscrivant son propre nom dans cette lignée d'aventuriers ou de géographes ayant la «passion du monde ».

\section{Le parcours du géographe}

Comme nous l'avons vu, le discours sur la pédologie occupe une place importante dans le récit; en revanche, le parcours du géographe en terre mexicaine reste en grande partie occulté. Il faut d'abord souligner le fait que l'excursion dans la vallée du Tepalcatepec, maintes fois évoquée au cours du récit, est totalement absente de la diégèse. Le chapitre intitulé « Adieu à l'Emporio » se termine sur ces mots : «Voilà. C'est fini. J'ai quitté la Vallée » (229). Comme on sait que Daniel se dirige vers la frontière nord, on est amené à penser qu'il va d'abord essayer de retrouver Lili, mais l'ellipse temporelle et spatiale concernant l'excursion dans la vallée du Tepalcatepec crée une absence de taille. On ne voit même pas le personnage s'aventurer vers le Paricutin. Les seules traces que nous avons sous les 
yeux se trouvent dans l'itinéraire donné en annexe et dans la carte schématique qu'il a tracée - qui est d'ailleurs davantage un plan qu'une véritable carte. Ces éléments, truffés de détails techniques («Sol tertiaire, arénique congloméré, vers Santa Ana calcique-arénique » 289), trouveraient davantage leur place dans un rapport destiné à une Société de géographie, puisqu'ils sont en relation directe avec la profession exercée par le jeune homme. Ce dispositif soulève des questions intéressantes au sujet de la lecture. Ce n'est pas comme dans La quarantaine (1995), où les extraits $\mathrm{du}$ Carnet $\mathrm{du}$ botaniste ponctuent les épisodes relatés tout en s'inscrivant harmonieusement dans le cours temporel. Ici, on peut se demander si tous les lecteurs consultent ce rapport de terrain. Accordent-ils la même attention au récit qu'aux deux objets présentés en annexe, à savoir l'itinéraire et la carte ? Il y a fort à parier que bien des lecteurs se contenteront de survoler les annexes, de les considérer uniquement comme des "pièces à conviction», servant avant tout à créer une impression de vraisemblance et à renforcer le caractère scientifique de la mission du géographe. Quant à ceux qui auront la curiosité de lire ces documents de près, iront-ils jusqu'à établir des liens entre la carte et l'Itinéraire du Paricutin à la vallée du Tepalcatepec ? Si l'on fait une lecture croisée de la carte et des notes de terrain, on peut s'amuser à reconstituer le parcours du voyageur à travers les lieux mentionnés. Certains passages permettent d'imaginer sa pénible avancée parmi les montagnes et les plateaux : « Marche difficile, terrain aride, éboulements, ravins bouchés par la végétation épineuse » (289). Les remarques brèves donnent une idée du niveau de difficulté de l'excursion. Une fois que le guide Salvador repart vers son village, le marcheur fait face à une grande solitude. Toute son attention est retenue par la nature du sol qu'il a sous les pieds.

Il est difficile par ailleurs de ne pas céder à la tentation de replacer le petit morceau de carte qui nous est donné à la page 291 dans une carte plus large, celle du territoire évoqué dans le récit. En consultant une carte de l'Amérique centrale il devient possible de revenir sur ses pas, de relire le texte en tentant de cartographier le récit et l'itinéraire à partir des toponymes inscrits sur la carte et parsemés tout au long du roman, de reconstituer autrement dit les allées et venues des différents personnages. La lecture du roman, de la carte et de l'itinéraire donne lieu dans ce cas à un véritable exercice de topographie.

\section{Toponymes et patronymes}

Du grec topos, «lieu» et de nomos, «nom», le toponyme lie intrinsèquement le langage et le lieu. Fait de langue et d'espace, ce type de signe génère une tension entre le géographique et le linguistique. Dans l'espace romanesque, une autre tension s'ajoute, qui met aux prises la référentialité et l'imaginaire, dans la mesure où les auteurs utilisent des toponymes existants tout en inventant parfois eux-mêmes des noms de lieux. Cette entreprise rejoint l'histoire de la cartographie, puisque l'acte de nommer les lieux a présidé à l'élaboration de chaque toponyme existant. Cela dit, dans la grande majorité des cas, son origine a fini par tomber dans $1^{\text {'oubli. }}{ }^{1}$

\footnotetext{
${ }^{1}$ Qui sait encore que Yellowknife, la capitale des Territoires du Nord-Ouest, au Canada, est une
} traduction de Couteau-Jaune, du nom de la nation déné-métis qui vivait à cet endroit? 
À l'inverse de la situation courante, le nom inventé dans la géographie imaginaire naît en l'absence d'un contexte géographique : il est donc vide, entouré de vide. L'aventure toponymique n'est pas déclenchée par la découverte d'un endroit ou par un intérêt marqué pour un lieu; celle-ci accompagne le développement d'un récit, aussi bien dans le cadre du processus d'écriture que dans celui de la lecture. Comme le signale Pierre Jourde, «[1]e toponyme inventé est appel à la découverte. Il ouvre par l'absence de son référent une béance que le livre va dans une certaine mesure entreprendre de combler » (199). Telle est la fonction du titre du roman Ourania : ouvrir un espace fictionnel qui vient se superposer à l'espace réel le temps d'une lecture. À la question de Jérôme Garcin : « Pourquoi avoir choisi le ciel à la terre ? », Le Clézio répond : « Pour le nom de cette muse de l'astronomie dont je voyais le portrait dans un livre de Victor Duruy. Peut-être aussi parce que c'est une femme... » (2006). Là encore, comme c'est très souvent le cas dans l'œuvre leclézienne, c'est « du livre [que sortent] des mots, des noms », pour reprendre la phrase déjà citée qui parlait d'Ouranos, le ciel étoilé. Associé à une image de femme, à l'une des Muses ayant Zeus pour père et Mnémosyne pour mère, le nom se transformera en toponyme le temps d'un roman. Comme c'est le seul toponyme inventé dans ce livre, il fait figure d'exception, une exception de taille cela dit, puisqu'il s'agit du titre.

L'hybridité de l'espace littéraire découle du fait que l'espace romanesque est constitué de localités vérifiables et de localités imaginaires. D'après Yves Baudelle, qui s'est intéressé à la toponymie et à l'onomastique, «aucun roman n'est réductible à des données empiriques ou mémorielles, aucun [n'est] non plus purement fictif ou verbal » (Cartographie réelle 47). Le toponyme possède à la fois une fonction référentielle et une fonction sémiotique : "Le modèle proposé ici est celui d'un feuilletage: à leur valeur référentielle les toponymes romanesques ajoutent des valeurs sémantiques, qui s'y superposent sans l'effacer. ... En somme, il s'agit d'introduire des courbes de niveaux dans la cartographie fictionnelle » (Noms de pays 50). Les romanciers ont plusieurs possibilités : ils peuvent inventer des toponymes ; transformer des toponymes connus, auquel cas « les noms propres ont une valeur d'indice » (50) dans la mesure où ils révèlent l'appartenance à une région ou à un pays, ce qui joue un rôle crucial dans l'illusion romanesque ; placer des bourgades fictives dans une région connue ; utiliser des toponymes du corpus cartographique, ayant une fonction référentielle; dans ce cas, le simple fait de les intégrer à une fiction a pour effet de les remotiver. En effet, à partir du moment où un toponyme participe à la cartographie romanesque, il s'intègre à un réseau et se charge d'un sens nouveau. Véritable interface entre le texte et le lecteur, il constitue un jalon pour la construction de l'espace romanesque, une piste alternative au récit qui se déroule quant à lui de manière linéaire. Le lecteur pourra selon les cas y percevoir des indices, capter un sens particulier à partir de ses expériences et connaissances, ou encore mettre en œuvre un véritable processus d'exploration.

Si l'on reprend la typologie proposée par Baudelle, ${ }^{2}$ on constate que mis à part le titre, Ourania, qui possède comme on l'a vu une fonction sémiotique

${ }^{2} 1$. Une fonction référentielle quasi exclusive (Noms de pays, de provinces, de grandes villes) ; 2 . Une fonction référentielle dominante (noms réels moins connus, vérifiables) ; 3 . Une fonction 
dominante, les toponymes du roman répondent majoritairement à une fonction référentielle. Les plus abondants sont ceux qui se rapportent au Mexique, plus particulièrement à l'état du Michoacan. L'ensemble est tellement détaillé et foisonnant qu'il serait possible de dresser une carte précise à partir des indications données au fil du récit. Mais en plus des lieux arpentés par le protagoniste, il faut tenir compte des toponymes relatifs à l'histoire des personnages secondaires, dont certains sont assez intrigants.

C'est le cas de l'hydronyme Canadian River, cité pour la première fois à la page 151: Jadi, le chef spirituel du village de Campos, est né à Konawa chez les Indiens Choctaws, près de la Canadian River. Des parallèles assez troublants peuvent être établis entre l'homme et la rivière. En premier lieu, il faut rappeler que la Canadian River servait de frontière entre les Indiens Choctaws et les Blancs ; il est vrai que ce rôle est généralement assigné aux cours d'eau (Hentsch). Or, Jadi a pour mère une femme appartenant à la nation diné (ou déné) et pour père un homme venu de Bordeaux. En tant que métis, il incarne la frontière culturelle; pour reprendre l'expression d'Amin Maalouf, c'est un " être de la frontière », dont le pendant géographique est un cours d'eau. En second lieu, le roman précise que Jadi a été élevé dans une autre réserve indienne, à Gallup en Arizona. Même si la ville de Gallup se trouve au Nouveau-Mexique, la réserve indienne dépasse les frontières entre les états américains et s'étale à la fois sur le Nouveau-Mexique et l'Arizona. Quand on trace la trajectoire de Jadi enfant, on s'aperçoit qu'elle suit trait pour trait une portion de la rivière. S'étendant sur plus de $1500 \mathrm{~km}$, celle-ci dépasse de loin certains fleuves, mais il s'agit bel et bien d'une rivière, plus exactement d'un tributaire du fleuve Arkansas. Toutes proportions gardées, la rivière et l'homme évoluent à l'unisson.

Pourquoi cette rivière se nomme-t-elle la « Canadian River»? En raison de son origine ? Intriguée par ce nom, j'ai cherché des cartes pour voir si en remontant la rivière on arrivait au Canada. Il n'en est rien. La rivière Canadienne prend sa source au Colorado et traverse ensuite le Nouveau-Mexique, le Texas et l'Oklahoma. À aucun moment elle n'entre en territoire canadien. C'est la piste de l'histoire qu'il faut remonter si l'on veut comprendre l'origine de cet hydronyme. Selon toute vraisemblance, celui-ci serait lié à la présence des Canadiens (Canadiens-français) qui ont exploré le continent nord-américain depuis les débuts de la Nouvelle-France et qui empruntaient régulièrement cette voie de navigation. À l'époque, le canotage et le portage constituaient en effet les moyens de transport les plus usités. Comme le rappelle Christian Morissonneau dans le collectif intitulé Franco-Amérique, cinq catégories de Français ont nommé le continent entre le $\mathrm{XVI}^{\mathrm{e}}$ et le $\mathrm{XIX}^{\mathrm{e}}$ siècles : les découvreurs et explorateurs comme Samuel de Champlain, qui a baptisé à lui seul pas moins de 300 lieux ; les traiteurs (ceux qui faisaient la traite des fourrures), autrement dit des trappeurs, des coureurs des bois qui prenaient la plupart du temps pour épouse une Amérindienne et qui adoptaient le mode de vie amérindien ; les missionnaires ; les officiers militaires au service du roi ; enfin, le groupe encore plus nombreux de « ceux qui s'établissent, défrichent,

sémantico-référentielle (noms fictifs, mais typiques, à connotation géographique); 4. Une fonction sémiotique dominante (noms forgés, dont la signification est feuilletée). (Noms de pays 59-60) 
commercent quand il le faut, rencontrent les Amérindiens là où ils s'installent ou vont au devant d'eux, comme engagés ou voyageurs, [ils] créent la société métisse de l'Ouest » (Nommer l'Amérique 31). À tous les toponymes d'origine française éparpillés à travers le continent nord-américain, parfois tellement transformés par l'usage et par l'imprégnation de la langue anglaise que toute trace de l'origine s'est perdue en chemin, il faut ajouter les toponymes créés par les anglophones pour désigner les lieux dans lesquels des «Francos», des «French», souvent métis, étaient installés :

Il est à noter que ceux qui ont nommé en français sont évoqués à leur tour par la toponymie anglophone. ... Lors de l'expédition de Lewis et Clark (18041806) qui traverse les États-Unis, de Saint Louis au Pacifique, tous les Canadiens engagés comme guides ou rencontrés sont des French ou des Frenchmen (Vaugeois, 2002). Pour une rivière Canadienne (Canadian River, New Mexico), existe au Minnesota, au Michigan, en Indiana ou au Dakota une foule de French City, Lake ou River. Alors sont confondus, surtout en raison de la langue, les Canadiens et les Français. En ce sens-là, et vue par les Anglo Americans, il y a bien une French America (32)

L'histoire officielle n'a pas conservé de traces de cette présence canadienne en terre américaine. Seuls les toponymes et les patronymes gardent encore en mémoire quelques bribes du passé. Le terme de «French » utilisé pour parler des Canadiens-français n'a-t-il pas donné lieu à des malentendus ? Les spécialistes de la Franco-Amérique s'accordent pour dire que les «Frenchmen », les Canadiensfrançais, se mariaient généralement avec des Amérindiennes. ${ }^{3} C$ 'est bien ce type d'union que contracte le père de Jadi, dont on sait très peu de choses à vrai dire. Le roman est très laconique à son sujet. De l'avis des géographes, il apparaîtrait beaucoup plus vraisemblable que le père de Jadi soit un «French », un « voyageur » canadien-français, plutôt qu'un Français de Bordeaux débarqué en Oklahoma à la fin du $\mathrm{XIX}^{\mathrm{e}}$ siècle ou au début du $\mathrm{XX}^{\mathrm{e}}$ siècle. Cela dit, un romancier a tous les droits lorsqu'il s'agit de créer un être de fiction, y compris celui de créer une cartographie décalée par rapport à l'histoire des lieux.

Une autre connexion unit le Nouveau-Mexique et le Canada dans Ourania étant donné que Jadi devient le père spirituel de Raphaël, un jeune homme originaire du Québec. Ce dernier apparaît d'ailleurs comme un double de Jadi, surtout si l'on observe sa trajectoire et son origine. Métis lui aussi, il a un père innu, amérindien, et une mère blanche, québécoise, alors que Jadi a un père blanc, français, et une mère amérindienne. Une inversion s'opère autrement dit entre les deux hommes. Quand Daniel croise Raphaël pour la première fois, ce dernier lui raconte qu'il est né à Rivière-du-Loup et que son père l'a conduit jusqu'à Campos après la mort de sa mère. Parvenu à l'âge où il peut quitter le village tout seul, il a décidé d'aller à Manzanillo, sur la côte ouest du Mexique : "Moi je voulais voir la mer, parce que j'ai oublié la mer depuis que j'ai quitté mon pays » (Ourania 28) Rivière-du-Loup est situé sur la rive sud du Saint-Laurent, en face de l'embouchure

\footnotetext{
${ }^{3} \mathrm{Au}$ Canada, ces unions sont à l'origine du peuple métis, dont Louis Riel est le représentant le plus illustre.
} 
du Saguenay. C'est encore assez loin de la mer, mais cet estuaire est l'un des plus larges du monde (il mesure $23 \mathrm{~km}$ à cet endroit), ce qui fait qu'à partir de Rivièredu-Loup les gens ont pris l'habitude de dire « la mer» plutôt que « le fleuve ». La toponymie reflète cette manière de voir le Saint-Laurent, puisqu' on y trouve, entre autres, le " Chemin de la mer », le " Chemin des marées », et un peu plus au nord, une localité nommée Métis-sur-mer. Le père de Raphaël est quant à lui originaire « du Lac Saint-Jean, au nord du Québec, une région où - nous dit le texte - il n'y a pas de routes, seulement des forêts et des rivières » (122). Ce petit écart par rapport à la géographie du Québec - il y a tout de même quelques voies d'accès dans cette région - passerait facilement inaperçu si on ne trouvait pas, une page plus loin, un élément assez incongru : on y apprend que le père de Jadi s'est cassé la jambe dans un "piège à élan » (122), qu'il a été soigné dans le village de Marthe, dont il est tombé amoureux, et qu'il est ensuite " reparti vers Saint-Jean » (123). Or, le terme d' " élan », employé couramment pour désigner les animaux de Scandinavie et de Sibérie, n'est pas du tout utilisé en Amérique du nord, où ce cervidé est connu sous le nom d'orignal. Par ailleurs, les pièges à élan ont été utilisés par les Autochtones avant l'arrivée des Blancs et de leurs fusils, soit plusieurs siècles avant que le père de Jadi ait son accident. À son époque, soit la première moitié du $\mathrm{XX}^{\mathrm{e}}$ siècle, la chasse à l'orignal se faisait au fusil ou à l'arc. L'impression d'un décalage avec la réalité québécoise est amplifiée par l'utilisation du toponyme « Saint-Jean » au lieu de «Lac Saint-Jean », ce qui crée une incohérence sur le plan cartographique. Il faut savoir en effet que la ville de Saint-Jean se trouve au Nouveau-Brunswick et non au Québec, à une distance d'environ $900 \mathrm{~km}$ du Lac Saint-Jean. Celui-ci possède les dimensions d'une véritable mer intérieure, mais son nom ne provient pas d'une ville ou d'un village situé sur ses bords. ${ }^{4}$

Une autre catégorie de toponymes renvoie aux îles et se trouve associée presque exclusivement aux personnages féminins : Hoatu vient de l'île de Raiaeta, dans le Pacifique, l'une des Îles Sous-le-Vent de la Polynésie française ; on sait peu de choses sur son passé, à part le fait que son père militaire l'a emmenée en Californie (172); devenue la guide de la tribu de Campos au moment de l'exil, son identité îlienne se renforce à la fin du roman puisqu'on la voit pour la première fois vêtue d'un paréo à la mode mahoie (263) en train de marcher « pieds nus, comme chez elle, à Raiaeta » (264). Dahlia, la petite amie de Daniel, provient de l'île de Porto Rico, où elle retourne d'ailleurs après un épisode au Mexique ; c'est dans cette île que prend fin le roman. Rosalba, la mère de Daniel, a un prénom des îles :

\footnotetext{
${ }^{4}$ Cela peut paraître curieux, mais quand on connaît un peu les usages en matière de toponymie au Québec, rien ne peut nous étonner. Ceux-ci ont en effet de quoi dérouter ceux qui ne sont pas tombés dedans quand ils étaient petits. Déjà, le fait d'utiliser un seul et unique nom - Québec - à la fois pour la province et pour sa capitale (qui n'est pas la plus grande ville de la province, contrairement à l'usage courant), rend perplexe la majorité des visiteurs. Cela se complique dès que l'on cherche par exemple à décrire le réseau des Universités du Québec. Il y en a à Montréal, à Trois-Rivières, à Hull, à Rimouski, à Chicoutimi, mais pourquoi n'y a-t-il pas d'Université du Québec à Québec, me direz-vous ? C'est que, dans la capitale, ou plutôt dans sa banlieue, à Lévis, se trouve l'Université Laval, la plus ancienne université de la province ; cela dit, il ne faut pas faire l'erreur de chercher l'Université Laval à Laval (la $3^{\mathrm{e}}$ ville en importance de la province), où se trouve plutôt un campus de l'Université de Montréal. Bref, comme on s'en aperçoit assez rapidement, les repères toponymiques sont assez énigmatiques.
} 
«Un nom doux et léger, un nom qui évoquait son île, qui allait avec son rire, ses chansons et sa guitare. Elle s'appelait Rosalba »(14). De quelle île s'agit-il ? Quand l'a-t-elle quittée ? Comment s'est-elle retrouvée dans la maison de ses beaux-parents ? Le roman ne nous le dit pas. Rosalba est associée à la lecture, à la Grèce : « ce gros livre que ma mère lisait, et qui parlait de la Grèce, de ses îles. ... C'est dans le livre que j'ai trouvé le nom du pays d'Ourania. C'est peut-être ma mère qui a inventé ce nom, pour partager mon rêve. » (18) Or, Rosalba n'est pas un prénom grec ; tout ce qui concerne son origine, ou sa trajectoire, reste dans la brume. Le nom du père, Alain Sillitoe, évoque quant à lui l'écrivain britannique Allan Sillitoe, ayant vécu à Majorque en Espagne (encore une île) avec son épouse Ruth Fainlight, une poétesse américaine.

Enfin, un autre phénomène reliant le lieu et l'écriture mérite d'être mentionné. Il se rapporte à un acte choronymique, acte par lequel on nomme un lieu (Morissonneau Le langage) : "On m'a raconté qu'autrefois, au temps des jésuites, vivait à Campos un homme qui avait nommé l'endroit Armen, ou Almen, ce qui signifiait pierre dans sa langue, parce qu'il n'y avait que des pierres ici. Et le nom est resté » (165). Si le nom est resté, en revanche un silence entoure la langue dont il est issu, une langue que l'on devine à partir de certains indices. Armen signifie «pierre" en breton, mais il n'est pas utilisé comme toponyme en Bretagne ${ }^{5}$; c'est plutôt le terme « roc'h » qui traduit la prégnance de la pierre dans le paysage armoricain (Roc'h Trevezel, Roc'h ar Vran...). Il ne semble pas qu'un lieu ait été nommé Armen dans cette région du Mexique - cela n'apparaît que dans les courbes de niveau du romanesque -, mais il est fort probable que des jésuites bretons s'y soient rendus. Si l'on en croit Raphaël, le toponyme se serait déplacé du lieu à la langue : employé pour désigner l'endroit, il a surtout servi par la suite à désigner la langue parlée à Campos, l'elmen. Un déplacement qui inaugure bien la malléabilité propre à cette langue : «Dans elmen, chacun parle comme il veut, comme cela lui vient, en changeant les mots, ou bien en se servant des mots des autres » (165). Le toponyme s'est-il transformé en nom de langue ou bien a-t-il fusionné avec la langue ? La préposition «dans elmen » laisse croire en effet à une intrication étroite entre les deux, comme si la langue, circonscrite à un lieu précis, s'était définitivement inscrite en lui.

Toponymes mexicains, canadiens, français, polynésiens, portoricains, bretons... le roman de Le Clézio construit une cartographie aux dimensions de la planète, en mêlant les idiomes pour le plus grand plaisir des lecteurs ayant la «passion du monde. » Afin d'évoquer l'ailleurs, le roman met à profit divers éléments : la vision propre à un géographe-voyageur, sa curiosité, son discours scientifique et son parcours à travers le Mexique, une carte et des notes de terrain, des toponymes, des patronymes, des prénoms donnant des indices de lieux lointains. Tous ces éléments mêlent étroitement le linguistique et le géographique, au point où certains personnages ont des destins liés à des cours d'eau, au ciel étoilé

${ }^{5}$ C'est le nom d'une revue bien connue, cela dit (ArMen) et celui d'un phare également, Ar-Men, situé sur l'île de Sein. 
ou aux îles. La diversité géographique se lit à travers la diversité des langues et des cultures, une véritable mosaïque à l'image de la communauté installée à Campos, où sont parlées plusieurs langues. Comme le souligne Thierry Léger, le village « constitue un véritable métissage culturel ». Loin de se vêtir d'une tonalité unique, aux couleurs mexicaines, l'ailleurs s'esquisse dans ce roman à partir de références très diversifiées, faisant naître un véritable chatoiement. Au point où l'on peut se demander si l'alliance du lieu et de l'écriture n'est pas, finalement, l'un des vecteurs privilégiés de l'imaginaire.

\section{Bibliographie}

Ballot, Pierre-Louis. « Ourania, de Jean-Marie Gustave Le Clézio : Quand le géographe-missionnaire devient un géographe-voyageur. » Journal Le Point G 5 (décembre 2011) : 6-7. Les cafés géographiques. Bordeaux : Université Michel de Montaigne Bordeaux 3. Web. 10 août 2016.

Baudelle, Yves. «Cartographie réelle et géographie romanesque : Poétique de la Transposition. » Cahiers de narratologie : Création de l'espace et narration littéraire 8 (1997) : 46-63. Lavergne, Gérard (dir.). Narratologie.revue.org. Web. 10 août 2016.

---. « Noms de pays ou pays des noms ? Toponymie et référence dans les récits de fiction. », Topographies romanesques. Camus, Audrey et Rachel Bouvet 
(dir.). Rennes/Québec : PUR/PUQ, 2011. 45-62. Imprimé.

Benvéniste, Émile. Problèmes de linguistique générale 1. Paris : Gallimard, 1966. Imprimé.

Berdoulay, Vincent. « La métaphore organiciste : Contribution à l'étude du langage des géographes. » Annales de géographie 507 (1982) : 573586. Imprimé.

Bouvet, Rachel. « Notes de traduction et sensation d'exotisme dans La trilogie de Naguib Mahfouz. » Revue de littérature comparée 3 (juillet-septembre 1997) : 341-365. Imprimé.

---. Vers une approche géopoétique : Lectures de Kenneth White, Victor Segalen et J.-M. G. Le Clézio. Québec : PUQ, 2015. Imprimé.

Bureau, Luc. Terra erotica. Montréal : Fides, 2009. Imprimé.

Cavallero, Claude. « L'utopie dans Ourania de J.-M. G. Le Clézio : Dilemme du rêve et du réquisitoire. " Mythe et création $2:$ L'œuvre, l'imaginaire, la société. Santi, Sylvain, Jean-Pol Madou et Laurent Van Eynde (dir.). Chambéry : Presses de l'Université de Savoie, 2007. 209-221. Imprimé.

Eprendre, Nicolas. Élisée Reclus : La passion du monde. Images de Rideau, Nicolas et Nicolas Eprendre. Son de Ruette, François-Xavier. Montage de Bouteiller, Christine. Antoine Martin Productions, Groupe Galactica, Vosges Télévision, 2012. DVD.

Hentsch, Thierry. La mer, la limite. Montréal : Héliotrope, 2006. Imprimé

Holtz, Grégoire et Vincent Masse. «Étudier les récits de voyages : Bilans, questionnements, enjeux. » Érudit.org/revue/arbo. Arborescences 2 (2012). Web. 24 août 2016.

Jacob, Christian. L'empire des cartes : Approche théorique de la cartographie à travers l'histoire. Paris: Albin Michel, 1992. Imprimé.

Jourde, Pierre. Géographies imaginaires de quelques inventeurs de mondes au XXe siècle: Gracq, Borges, Michaux, Tolkien. Paris : José Corti, 1991. Imprimé.

Le Clézio, J.-M. G. Ourania. Paris : Gallimard, 2006. Imprimé.

---. La quarantaine. Paris : Gallimard, 1995. Imprimé.

---. L'Inconnu sur la terre. Paris, Gallimard, 1978. Imprimé.

---. "Voyage en utopie. Entretien avec Jérôme Garcin.», Le nouvel observateur

2152, 2-8 février 2006. 88. Imprimé

Léger, Thierry. « Les paradoxes du métissage culturel et littéraire. » J.-M. G. Le Clézio : Dans la forêt des paradoxes. Moser, Keith et Bruno Thibault (dir.). Paris : L’Harmattan, 2012. 263-271. Imprimé.

Lévy, Bertrand. « Géographie et littérature : une synthèse historique. » Le GlobeRevue genevoise de littérature : Géographie et littérature 146 (2006) : 2552. Imprimé.

Maalouf, Amin. Les identités meurtrières. Paris : Grasset, 1998. Imprimé. Morissonneau, Christian. « Nommer l'Amérique. » Franco-Amérique. Louder, Dean et Éric Waddell (dir.). Québec : Septentrion, 2008. 27-40. Imprimé.

---. Le langage géographique de Cartier et de Champlain: Choronymie, vocabulaire et perception. Québec : Presses de l'Université Laval, 1978. Imprimé. 
Moura, Jean-Marc. «L'exotisme fin de (XXe)-siècle. » Revue de littérature comparée 296.4 (octobre-décembre 2000) : 533-553. Imprimé.

Rondeau, Daniel. « Ourania : le ciel de le Clézio. » l'express.fr/culture/livre. L'Express, 16 février 2006. Web. 10 août 2016.

Roussanne, Albert. L'homme suiveur de nuages, Camille Douls, Saharien (18641889). Introduction de Le Clézio, J.-M. G. Rodez : Éditions du Rouergue, 1991. Imprimé.

Roussel-Gillet, Isabelle. « Les cartes du ciel à l'œuvre chez Le Clézio. » Revuetextimage.com. Textimage : Cartes et plans 2 (été 2008). Web. 10 août 2016.

Salles, Marina. «Ourania de J.-M. G. Le Clézio : une utopie historisée, un roman politique. » Seer.fclar.unesp.br. Itinerários 32 (2011) : 127-142.

Araraquara : UNESP. Web. 10 août 2016.

Segalen, Victor. Essai sur l'exotisme : Une esthétique du divers. Montpellier : Fata Morgana, 1978. Imprimé.

Thibault, Bruno. «L'horizon fabuleux et l'écriture de la fuite dans Hazaran et Ourania de J.M.G. Le Clézio. » Horizons Lecléziens. Bernabé, Maria Luisa (coord.). Toulouse : Inter-Lignes, 2009. 117-26. Imprimé.

Van Acker, Isa. « Ourania : petite victoire sur fond de débris. » Cahiers Le Clézio 1 (2008) : 174-178. Imprimé.

Waddell, Éric. «L'œil géographique. », Au rythme des vents et marées... Morisset, Jean et Éric Waddell (dir.). Montréal : La Traversée-Atelier québécois de géopoétique. Carnets de navigation 1 (2005) : s. $p$. fr.calameo.com. Web. 10 août 2016.

White, Kenneth. Le plateau de l'albatros: Introduction à la géopoétique. Paris : Grasset, 1994. Imprimé. 